\title{
Nonlinear Noise Estimation in International Capital Markets
}

\author{
Costas Siriopoulos \\ University of Macedonia, Greece \\ Alexandros Leontitsis \\ University of Kent at Canterbury, U.K.
}

\begin{abstract}
We analyzed six stock exchange markets through the nonlinear dynamics concept. We used daily data from the Toronto Stock Exchange, NYSE, London Stock Exchange, Hong Kong Stock Market, Tokyo Stock Exchange, and the Singapore Stock Exchange. The period studied is from January 1, 1988 to June 30, 1999. We performed Local Principal Components Analysis in order to estimate the dimension of each underlying attractor. Our main interest is the noise level estimation of each time series. The results indicate weak determinism and strong noise influence. The noise-to-signal ratio for almost all time series is above $50 \%$. Noise is leptokurtic in the eastern stock markets, and mesokurtic in western ones. (JEL C22, G15).
\end{abstract}

Keywords: chaos theory, local principal components analysis, noise estimation, nonlinear dynamics.

\section{Introduction}

In recent years, the development on nonlinear science has had a great influence in the analysis of the financial time series. Under this influence, many financial economists and researchers treated the financial markets as nonlinear dynamical systems with important implications in the Efficient Market Hypothesis (EMH), capital market

\footnotetext{
${ }^{*}$ We would like to thank Professor J. A. Scheinkman for providing us with the program of BDS statistic, and Professor E. Pilotte for his constructive comments in a previous version of this paper. We also thank two anonymous referees of the Multinational Finance Journal for their helpful comments and suggestions. An earlier version of this paper was presented at the $7^{\text {th }}$ Annual Conference of the Multinational Finance Society, April 5-8, 2000, Philadelphia, USA.
}

(Multinational Finance Journal, 2002, vol. 6, no. 1, pp. 43-63)

(C) Multinational Finance Society, a nonprofit corporation. All rights reserved.

DOI: $10.17578 / 6-1-3$ 
integration, and modern financial risk management.

Observed nonlinear behavior may be due to a number of reasons: the microstructure character of the market itself, the "herding behavior" of the investors, the regulatory framework involved, the low frequency of information input in the market relative to the observed prices, the market anomalies, and the existence of transaction cost. ${ }^{1}$ In addition, not all the participants in the market are equal in their ability to process information (see Heiner [1983], Kaen and Rosenman [1986]). ${ }^{2}$

While several researchers have reported evidence of non-IID behavior and nonlinear dependence in financial time series (i.e. Abhyankar, Copeland, and Wong [1995], Cecen and Erkal [1996], Hsieh [1989], Scheinkman and LeBaron [1989]), utilizing several statistical tests, few of them argue that a market is a strictly deterministic dynamical system (i.e. Blank [1991], Mayfield and Mizrah [1992]). ${ }^{3}$ In other words, they do not provide us any information about the stochastic part of the time series and its characteristics. In this study we assume that financial markets behave as stochastic dynamical systems with elements of both deterministic and stochastic behavior.

The dynamical models can be represented by differential (continuous) or difference (discrete) equations. Difference equations allow for a greater variety of approaches to be used to model the mapping from past values to the present one, parametric, neural networks, non-parametric, and local linear models (see Timmer [1998]). For all these methods a significant amount of observational noise can be a severe problem. Especially for difference equations the functional relation between past and present values will be underestimated if

1. The existence of transaction costs influence investors by reacting to arrival of news with delay, until verification of news is available. Furthermore, there is by nature, a difference in the attitude of investors towards positive and negative news. This is quite close to the theory stating that investors are loss averse rather than risk averse. Hence, the negative news tends to be overvalued relative to the good news.

2. According to the theory advanced by Heiner (1983), market participants differ in their ability in making the correct buy and sell decisions under uncertainty. Kaen and Rosenman (1986) showed that the spread between the noise traders and the experienced traders in conjunction with the complexity of the information, results in price changes in the same direction and price change behavior appears to be in non-periodic cycles.

3. The rejection of the hypothesis that stock returns are IID does not contradict market efficiency, and does not provide evidence of chaos in the stock market under study. However, the rejection of IID is consistent with the view that stock market returns are generated by nonlinear stochastic systems. It is also consistent with the presence of low complexity chaotic behavior in stock returns (see Hsieh [1991]). 
observational noise is not included in the model, and the degree of underestimation depends on the signal-to-noise ratio. However, stock price movements are discrete: there are lower bounds on the minimum observed nonzero price movement and indivisibilities in the observed price series (Shaffer [1991]). Furthermore, the econometric methods about the discrete time approach are far more developed than their continuous time counterparts.

The most common way used to investigate whether a time series is chaotic, involves the so-called "correlation dimension" technique originally introduced by Grassberger and Procaccia (1983). In short, if the correlation dimension saturates to a positive finite value, the time series is considered chaotic (some exceptions occur if the time series has strong serial linear correlations). In many cases of chaotic attractors, the correlation dimension does not saturate due to the presence of noise and has an upward trend, thus suggesting purely stochastic behavior (i.e. DeCoster et al. [1992], Eldridge, Bernhardt, and Mulvey [1993], Frank and Stengos [1989], Willey [1992]).

However, the influences derived by the direct application of nonlinear methods in finance and economics are affected by a number of problems. As noted by Hsieh (1992), Guillaume (1995), and Ramsey et. al. (1990), relatively short data sets make the significance of the results ambigous. Also, following Szpiro (1997), Guillaume (1995), Savit (1988), and Casdagli and Eubank (1992), price fluctuations are dominated by noise, and one important goal of the analyst is then to understand the precise nature of the noise. Many economic forces act similar to stochastic shocks. This means that economic and financial processes are "contaminated" with random noise which makes the task of discriminating (if present) intrinsic chaos much more difficult. In practice, the existence of noise is the cause of the difficulty in distinguishing between the deterministic and stochastic processes (Mirowski [1990]). Therefore, the detection of noise can lead us to a better understanding of the true financial time series dynamics, let us model these time series in a better way, and finally improve the forecasting. Savit (1988) also notes: "using the ideas and the framework introduced (i.e. nonlinear dynamics), it should be possible to improve price predictability and to find general patterns in price movements that heretofore have been largely regarded as random". Thus, it is very important to estimate the noise in financial time series, as a first step to a better understanding of the financial time series under the nonlinear dynamics concept. In this study we will discuss a methodology for 
separating noise from chaotic processes.

The rest of the study is structured as follows: Section 2 addresses the recent literature on the subject and the motivation of this study. In section 3 we describe our data sets. Section 4 introduces the reader to the methodology used, with the main results presented in section 5 . Finally, section 6 concludes.

\section{Recent Literature and Motivation}

There is a growing body of literature applying the concepts of nonlinear dynamics and chaos theory to the field of economics and finance. ${ }^{4}$ Savit $(1988,1989)$ provides an analytical framework for applications of chaos to asset prices and in particular to options prices, while Hsieh (1991) discusses other methological issues in detecting chaotic and nonlinear behavior in stock market returns.

Brock and Sayers (1988) found nonlinearity in the U.S. labor market and investment. Barnett and Chen (1988) discovered low dimensionality in some U.S. monetary measures, while Frank et.al (1988) indicated nonlinearities in Japan's real GDP. Scheinkman and LeBaron (1989), Ashley and Patterson (1989) also report evidence of nonlinearities and chaotic behavior testing CRSP data. In addition, De Grauwe et.al. (1995), Hsieh (1989) applying similar techniques found strong nonlinear dependencies in a number of foreign exchange rate returns, albeit weak chaotic behavior.

Although the existence of nonlinear dependencies and chaotic behavior is well documented in financial time series, the presence of noise weakens the forecasting ability of the applied models. Jaditz and Sayers (1995) found that chaotic predictions were not significantly better than $A R(p)$ predictions, while in the case of Diebold and Nason (1992) random walk predictions were comparable with chaotic predictions. Thus, there is empirical evidence that noise has a strong influence on financial time series. On these grounds Guillaume (1995) utilized other techniques for noise reduction and reported the absence of low-dimensional attractor for the foreign exchange markets. Szpiro (1997) in studying the S\&P 500 Index calculated the correlation integral by the method of hyperspheres, and found an increasingly presence of

4. Baumol and Benhabib (1989) give a good survey of economic models, which produce chaotic behavior. See also Baumol and Quandt (1985). 
noise.

The most common way to estimate the noise level of a financial time series is the residuals' norm of some stochastic model. A common choice is the GARCH model, which has the advantage of dealing with heteroskedasticity. However, there are two serious disadvantages of this choice. The first one has to do with the simplicity of dynamics of that model, considering the complexity of the financial time series behavior. There are many references that indicate nonlinear structure on financial time series, which cannot be extracted with a GARCH model (Yang and Brorsen [1993]). The second one has to do with the noise amplification through the dynamics of the model.

The solution to the above problems comes from the nonlinear dynamics concept. First, we are able to use complicated models, such as the local linear models. These models consider that every cluster (or neighborhood) of points is a special case because it has its own dynamics (approximated linearly). Second, we do not utilize the dynamics of each neighborhood in order to estimate the noise level. Therefore, we do not amplify the noise through our model's dynamics. We can estimate noise geometrically, i.e. by measuring the deviation of each neighborhood from smoothness. A noise-free time series is considered as the result of a system whose successive values are lying on a smooth hypersurface. Therefore, any deviation from this smoothness is considered noise.

This study extends the previous literature applying a new concept on estimation of the standard deviation of the noise. The aim is to utilize modern nonlinear techniques in order to conclude about the influence of noise, its characteristics, and its impact on the statistical properties of the financial time series. In particular, the presence of noise is explicitly taken into account and the methodology is applied to a large data set constisting of six international stock exchanges.

The methodological approach used follows five steps. In the first step, we apply the BDS statistic to test for independence, and the Variance Ratio statistic to test for a random walk. In the second step we determine the upper bound of the dimension of each time series with the Local Principal Component Analysis. We use this upper bound in a third step to estimate the correlation integrals from each time series, and in the next step we calculate the standard deviation of noise from their distortions. Finally, in the fifth step, we calculate the noise to signal ratio. The results indicate the presence of random noise in all six stock exchanges, supporting the market efficiency hypothesis. They also 
indicate that short-term prediction is equally hard for all the markets under study.

\section{Data Description and Preliminary Diagnostics}

The daily $\log$ returns of six international stock markets are analyzed. These markets examined are the Canadian (TSE 300 Composite), the US (S\&P 500), the UK (FTSE 100), the Hong Kong (Hang Seng), the Japanese (Nikkei 225) and the Singaporean (Straits Times). The first three markets are western and the last three are eastern. The period under study is from $1 / 1 / 1988$ to $30 / 6 / 1999$. That means we have eleven and a half years of daily data or 2900 observations for each data set.

The outliers for each data set were deleted bounding the time series from the western markets to $\pm 2 \%$, and the time series from the eastern markets to $\pm 4 \%{ }^{5}$ The descriptive statistics for each data set are given in table 1, Panel A.

The aim of the BDS test is to distinguish between IID data and any kind of dependence. This test is chosen because it can detect many types of departures from independence and identical distribution, and can serves as a general model specification test, especially in the presence of nonlinear dynamics.

The BDS statistic is asymptotically distributed as $N(0,1)$ under the null hypothesis of IID data, providing that the number of observations tends to infinity. ${ }^{6}$ It utilizes the correlation integral $C_{m}(r, T)$ defined in equation 6 , which has to be calculated for various embedding dimensions $(m)$. Let's define the $C_{1}(r, T)$ correlation integral for $m=1$. Brock, Dechert, and Scheinkman (1987) propose the BDS statistic:

$$
W_{m}(r, T)=\sqrt{T} \frac{C_{m}(r, T)-C_{1}(r, T)^{m}}{\phi_{m}(r, T)} \stackrel{d}{\longrightarrow} N(0,1)
$$

5. It is true that the wins oring scheme used is arbitrary and rather simplistic. However, the observations winsorized were few and mostly occurred on very active days. Moreover, other informal data winsoring schemes were used (based on thresholds or $z$-scores) and the results were found to be relatively insensitive.

6. Brock, Hsieh, and LeBaron (1991) report extensive simulations and show that the asymptotic distribution is a good approximation of the finite sample distribution when there are more than 500 observations. 
TABLE 1. Sample Characteristics

\begin{tabular}{|c|c|c|c|c|c|c|}
\hline & CAN & USA & UK & HK & JAP & SIG \\
\hline \multicolumn{7}{|c|}{ Panel A: Descriptive Statistics } \\
\hline No of Obs. & 2902 & 2905 & 2905 & 2869 & 2866 & 2857 \\
\hline Average & .0004 & .0007 & .0005 & .0008 & -.0001 & .0004 \\
\hline St. Dev. & .0063 & .0080 & .0082 & .0144 & .0133 & .0115 \\
\hline Skewness & -.2657 & -.0696 & -.0584 & -.1065 & .0194 & .0247 \\
\hline Kurtosis & 1.2774 & .3866 & -.0398 & 1.7066 & 1.1959 & 2.4082 \\
\hline Maximum & .0200 & .0200 & .0200 & .0400 & .0400 & .0400 \\
\hline Minimum & -.0200 & -.0200 & -.0200 & -.0400 & -.0400 & -.0400 \\
\hline \multicolumn{7}{|c|}{ Panel B: BDS test results } \\
\hline 2 & $13.18^{*}$ & $5.43 *$ & $3.99 *$ & $12.42 *$ & $14.43 *$ & $18.28 *$ \\
\hline 3 & $14.97 *$ & $7.37 *$ & $6.63 *$ & $15.65^{*}$ & $18.68^{*}$ & $21.65^{*}$ \\
\hline 4 & $16.01 *$ & $8.72 *$ & $8.42 *$ & $18.65^{*}$ & $21.95^{*}$ & $23.73 *$ \\
\hline 5 & $17.03^{*}$ & $11.05^{*}$ & $9.71 *$ & $21.37^{*}$ & $26.44 *$ & $25.78^{*}$ \\
\hline
\end{tabular}

Note: Panel A presents the descriptive statistics for each data set. Kurtosis coefficient is centered on zero. Panel B presents the BDS test results for each data set using $r$ equal to one standard deviation of the corresponding time series. For all countries and the period under study these results are significantly different from 0 . The hypothesis of IDD data can be rejected with a large probability. The asterisk $(*)$ means that the estimated result is significantly different from 0 at $5 \%$ level.

where $T$ is the total points on the phase space, $t \rightarrow \infty$, and $\phi_{m}(r, T)^{7}$ is an estimator of the asymptotic standard deviation of the $C_{m}(r, T)-C_{1}(r, T)^{m}$. If $\left\{x_{t}\right\}$ were IID, then $C_{m}(r, T)=C_{m}(r, T)^{m}$ (null hypothesis). ${ }^{8}$

7. The calculation ot the standard deviation is given by:

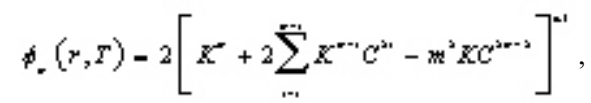

where $C-C_{1}(r, T)$ and,

$$
K=K(r, T)=\frac{6}{T(T-1)(T-2)} \sum_{i<\beta_{\alpha}} H\left(r-\left|x_{f}-x_{j}\right|\right) H\left(r-\left|x_{s}-x_{k}\right|\right) \text {. }
$$

See also Willey (1992)

8. Note that $C_{m}(r, T)=C_{1}(r, T)^{m}$ does not imply IID. BDS (1987) have shown that tests based on the $W$ statistic have a higher power for tests of stochastic or chaotic independence than other statistical techniques. Hsieh (1991) shows that BDS has a good power to detect at least four types of non-IID behavior: linear dependence, nonstationarity, chaos, and nonlinear 
The BDS statistic for each data set is given in table 1, Panel B. The critical value for the test is 1.96 , with a 5 percent level of significance. The results indicate significant dependence in all embedding dimensions. The results strongly reject the null hypothesis that stock returns are IID, since the values of the BDS statistic is much greater than $|2.0|$ in all cases. ${ }^{9}$ The large values for the BDS statistic in all cases but USA and UK, indicate strong evidence for nonlinearity in the data.

The Variance Ratio Test (Lo and MacKinley [1989]) was designed to recognize the random walk (null hypothesis) from any other process (alternative hypothesis). It can give us supplementary information about our time series. Under the random walk hypothesis, the variance of the $q$-differenced series $\left\{p_{t}-p_{t-q}\right\}$ is $q$ times the variance of the first differenced series $\left\{p_{t}-p_{t-q}\right\}$, where $p_{i}$ is the logarithm of the raw (price) time series. The variance ratio test is calculated for different values of $q$ for the six stock exchanges. For most countries the hypothesis of random walk is under question. The variance ratios generally increase with $q$ implying positive serial correlation for multiperiod returns. For USA and UK the variance ratios generally decline implying negative serial correlations for multiperiod returns. ${ }^{10}$

\section{Local Analysis}

In order to reconstruct the phase space of a dynamical system, given a time series $\left\{x_{i}\right\}_{i=1}^{N}$, we first have to choose an embedding dimension and a time delay $(\tau)$. Then we construct the vectors $x_{i}=\left(x_{i}, x_{i+\tau}, \ldots, x_{i+(m-1) \tau}\right)$, for $i=1,2, \ldots, T$, where $\mathrm{T}=N-(m-1) \tau$.

stochastic processes.

9. This result does not contradict market efficiency. Market efficiency implies that forecast errors of returns a re not predictable. The fact that returns themselves are not IID (and therefore potentially predictable) says nothing about the predictability of forecast errors. The rejection of IID is consistent with the view that stock returns are non-stationary, and also consistent with the presence of low complexity chaotic behavior in stock returns (Hsieh [1991]).

10. According to our results, Canada and Singapore do not follow the random walk with large probability. On the other hand, Japan and Hong-Kong are are consistent with the random walk hypothesis. Finally, for the U.K. and U.S.A. we obtained mixed results. 
A common criterion for the choice of $\tau$ is to take time delay equal to the time when the first minimum of mutual average information occurs (Fraser and Swinney [1986]). According to this criterion we calculate the mutual information of the data set before and after the time delay, and we choose the value of $\tau$ that first minimizes the mutual information. In our cases this first minimum can be relaxed since the value of mutual information drops sharply and saturates to a very small value for $\tau=1$ and beyond. So for all our data sets the mutual average information criterion indicated $\tau=1 .{ }^{11}$ This choice of $\tau$ is also the usual choice found in the literature for the financial time series.

If $d$ is the dimension of the attractor, we need $m \geq 2[d]+1$ to reconstruct the attractor successfully, where $[d]$ indicates the smallest integer larger than or equal to $d$ (Takens [1981]). The above condition was relaxed by Sauer, Yorke, and Casdagli (1991) to $m>2 d$.

The Local Principal Components Analysis (LPCA) (Broomhead, Jones, and King [1987]) can provide us by a rough estimation of $d$ even if large amount of noise distorts the phase space. The methodology of LPCA is the following.

The neighborhood of $x_{i}, U_{i, r}$, is defined by the points $\mathrm{x}_{j}$ whose distance from $x_{i}$ is less than or equal to $r$.

We define the neighborhood matrix $A_{i}$ to be:

$$
A_{i}=\frac{1}{\sqrt{\left|U_{i, y}\right|}}\left[x_{j}-x_{i}\right],
$$

where $\left|U_{i, r}\right|$ is the total points contained in the neighborhood $U_{i, r}$.

The elements of $A_{i}$ define an ellipsoid in m-dimensional phase space. The $D$ principal components (semi-axes) (where $D=[d]$ ) of this ellipsoid, which scale linearly with $r$, are the signal space, while the rest $m-D$ ones are the noise space. The curvature of the neighborhood is most contained in the noise space. The semi-axes of the ellipsoid are calculated via Singular Value Decomposition (SVD) algorithm (Press et al., [1996]).

More precisely, the SVD algorithm makes the decomposition $A=$ $G \bullet \Sigma \cdot V^{T}$ where $G$ is an orthonormal matrix $|U| \times m$ that contains the first $m$ eigenvectors of $A \bullet A^{T} ; \Sigma$ is a diagonal matrix $m \times m$ with the eigenvalues of $A^{T} A$ (also known as singular values of $A$ ), and $V$ is also

11. The diagrams are available upon request from the authors. 
an orthonormal matrix $m^{\prime} m$ with the eigenvectors of $A^{T} A$.

We are interested in the singular values of $A_{i}\left(\sigma_{1, i} \geq \sigma_{2, i} \geq \ldots \geq \sigma_{m, i}\right)$, which are the lengths of the neighborhood ellipsoid semi-axes. Finally, we average the $\mathrm{m}$ singular values of each neighborhood over all neighborhoods:

$$
\sigma_{h}=\left\langle\sigma_{h, i}\right\rangle, \text { for } h=1,2, \ldots, m,
$$

and plot $\log \sigma_{h}$ versus $\log r$.

The next and most important step of our analysis is the noise estimation. We will apply Schreiber's method (1993) as it was proposed for the noise estimation of chaotic time series. Our assumptions about noise are: (a) random uncorrelated fluctuations, (b) zero mean and constant variance, (c) with no other effect on the dynamics of the time series, (d) and a Gaussian-like symmetric distribution. ${ }^{12}$ The correlation integral (see Grassberger and Procaccia [1983]) denotes the fraction of the pairs of points that are included in a hypercube of size $2 r{ }^{13}$

$$
C_{m}(r, T)=\frac{2}{T(T-1)} \sum_{i<j} H\left(r-\left|x_{i}-x_{j}\right|\right)
$$

where $|x|$ is the maximum norm

$$
\begin{aligned}
& H(x)=1 \text { if } x \geq 0 \\
& H(x)=0 \text { if } x<0
\end{aligned}
$$

The derivative of $\log C_{m}(r, T)$ with respect to $\log r$, for small $I$, denotes the correlation dimension :

12. We can only recognize noise with constant variance, although the effect of heteroskedasticity is present to all of our time series. Filtering (even linear filtering) must be avoided (Badii et al. [1988], Broomhead, Huke, and Muldoon [1992], Theiler and Eubank [1993] because it increases the complexity of the time series and in some cases it can alter significantly its essential characteristics. In addition, noise is assumed to be Gaussian and additive (the non-Gaussian noise and its estimation problems is discussed in Timmer (1998).

13. Theiler (1986) corrected the correlation integral (see equation 6) for highly correlated data. According to his correction when the data are highly correlated (i.e. first autocorrelation above 0.9 ) we must ignore the neighboring in time values, because they will add some bias to the correlation integral. In our cases the first autocorrelation is relatively small, therefore there is no need to adopt this criterion. For the case of Canada is 0.215 , for USA 0.034 , for UK 0.077, for Hong Kong 0.079, for Japan -0.001 , and for Singapore is 0.187 . 


$$
d=\frac{d \log C_{m}(r, T)}{d \log r}
$$

Small fluctuations of $d$ are expected due to the presence of noise; therefore, $d$ becomes $d(r)$. When $m \geq d$ one expects $d(r) \approx d .{ }^{14}$

Let $k$ be another value for the embedding dimension with $k>m$. Schreiber (1993) proved that:

$$
\frac{d_{k}(r)-d_{m}(r)}{k-m}=g\left(\frac{r}{2 s}\right)
$$

where

$$
g(z)=\frac{2 z \exp \left(-z^{2}\right)}{\sqrt{\pi} \operatorname{erf}(z)},
$$

$\operatorname{erf}(x) \quad$ is the error function

$s \quad$ is the standard deviation of noise

$d_{k}(r) \quad$ and $d_{m}(r)$ are correlation dimensions corresponding to $k$ and $m$ embedding dimension respectively

For simplicity, we define $d_{k, m}(r)$ as the left-hand side of equation 6 . The value of $s$ must ensure the equality of equation 6. Using Brent minimization (see Press et al. [1996]) we can fit the theoretical model of the right-hand side of equation 7 to the experimental results of $d_{k, m}(r)$ and compute the error. $s$ is determined as:

$$
\min _{s}\left[\sum_{k}\left[d_{k_{, M}}(r)-\frac{r \exp \left(-\frac{r^{2}}{4 s^{2}}\right)}{s \sqrt{\pi} \operatorname{erf}\left(\frac{r}{2 s}\right)}\right)^{2}\right]
$$

where $s$ is a positive real value.

This method for noise estimation has the advantage that $d_{k, m}(r)$ reveals the noise distribution, which in many cases is not strictly

14. For a theoretical background on this concept see Sauer, Yorke, and Casdagli (1991) and for an experimental application see Ding et al. (1993). 
normal.

Finally, it is easy to estimate the Noise-to-Signal Ratio (NSR). This ratio is defined as follows:

$$
\operatorname{NSR}=\frac{s^{2}}{S^{2}} \approx \frac{s^{2}}{\hat{S}^{2}}
$$

where $S$ is the standard deviation of the noise-free time series, and $\hat{S}$ is the standard deviation of the noisy time series. Since we do not know the exact value of $S$ to estimate the NSR, we use $\hat{S}$ instead.

\section{Empirical Analysis and Discussion of the Results}

LPCA is used for the dimension calculation and Schreiber's method for the noise standard deviation calculation.

We start with LPCA in $m=5$ dimensions (figure 1). Guillaume (1995) applied the global SVD method and used a very large value for $m=50$. In this study we use the local SVD method and we set a sufficient embedding dimension $(m=5)$, which is consistent with the dimension that characterizes the low-dimension chaos.

All of our data sets seem to be one-dimensional because there is only one significantly large singular value that scales linearly with $r$. The remaining four singular values have approximately the same behavior. This is an evidence of determinism in each time series. However, the determinism seems to be weak for each index due to relatively large amount of noise. Recalling the results of BDS statistic and the variance ratio test statistic, we conclude in favor of a deviation from IID, and in rejecting the random walk, respectively.

In figure 2, we have the noise estimation results by the application of equation 7 with embedding dimension $k=3,4,5$ and embedding dimension $m=2$ (see equation 6). The radious $r$ is taken as a function of the range between the minimum and the maximum returns of each series (see table 1). As it is expected, there is an inverse relation between the estimated noise level and the calculated BDS statistic. Then we calculate the NSR's for the six different series (see equation 8). 

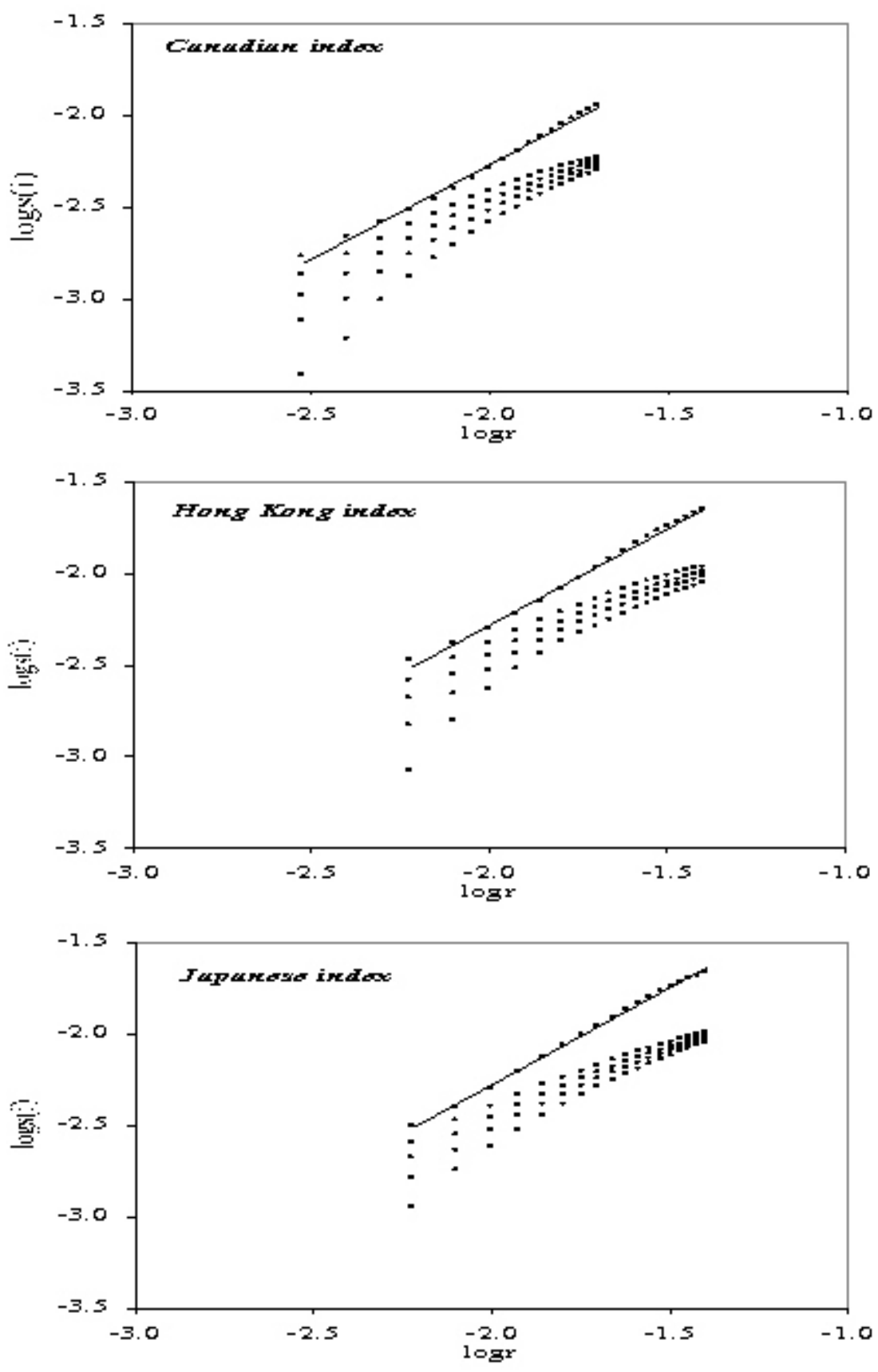
Multinational Finance Journal
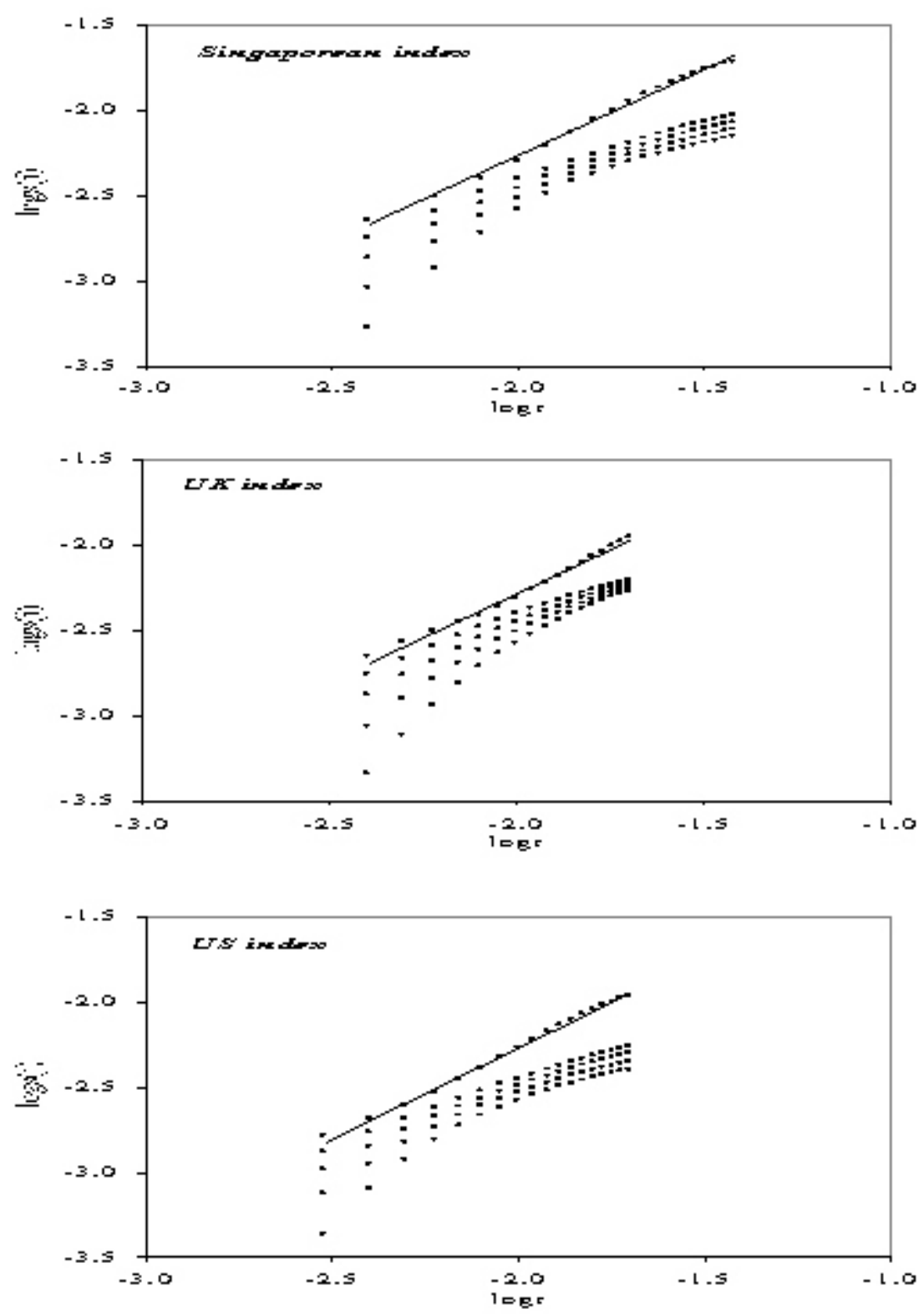

FIGURE 1.-LPCA for the six indices. We set $m=5$. The common characteristic of all these graphs is that only $\log \left(\sigma_{l}\right)$ scales linearly with $\log r$. Thus, we conclude that all time series are of dimension at most 1 . 

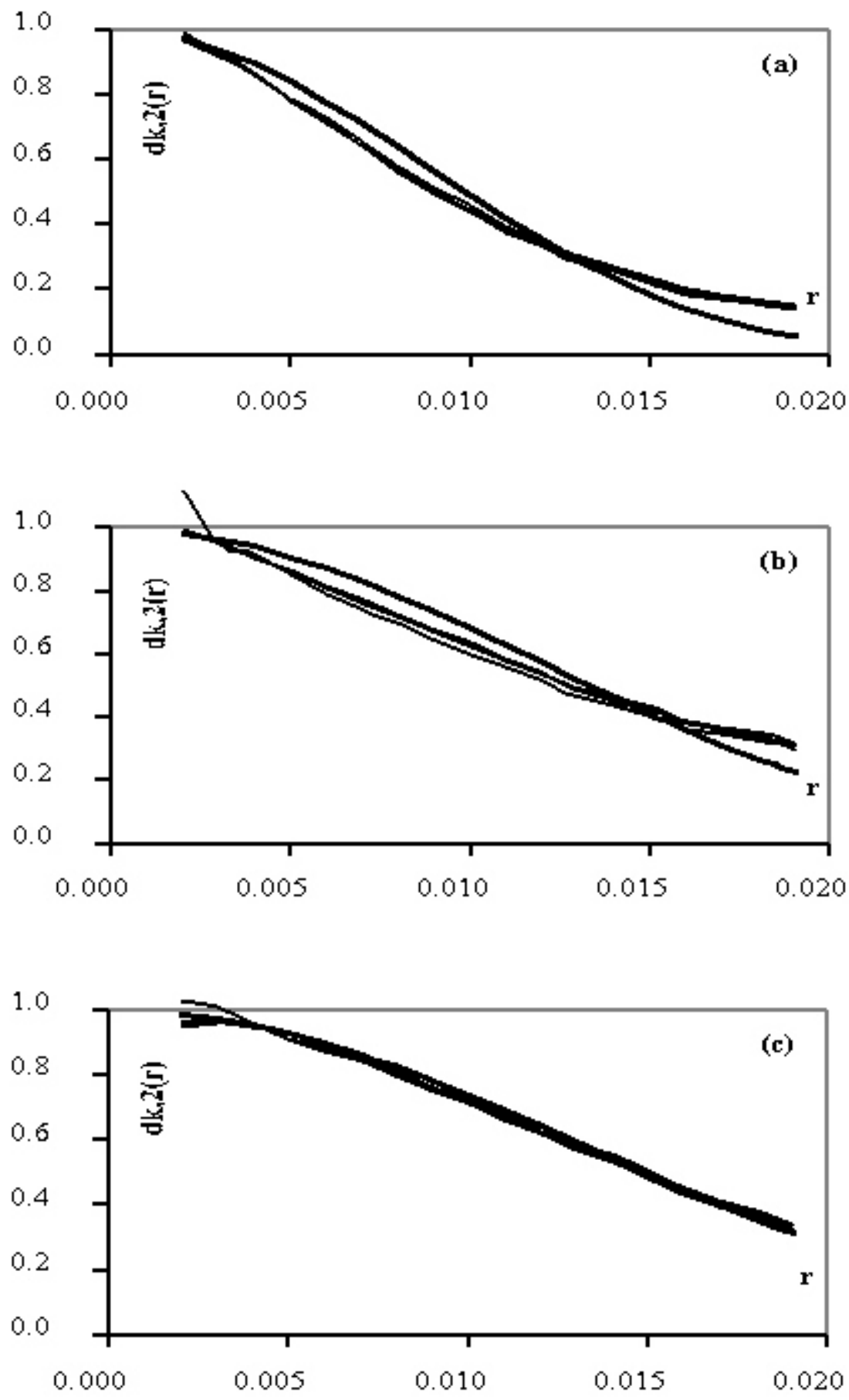

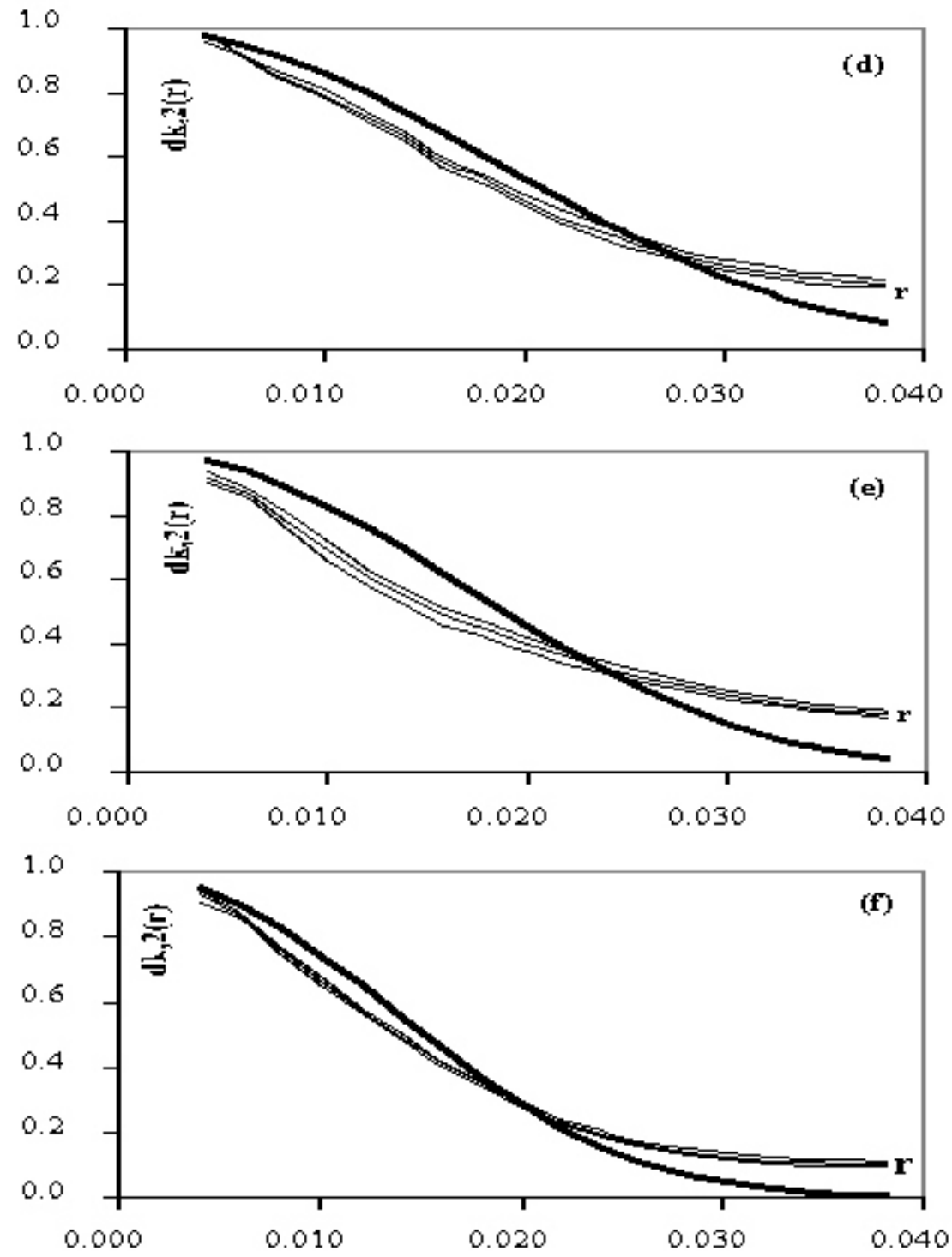

FIGURE 2.- Noise estimation for the six indices. The bold line indicates the curve we would observe if the noise were normal with mean 0 and the estimated standard deviation. (a) Canadian index. Estimated $s=0.0050, \mathrm{NSR} \approx 62.6 \%$. (b) US index. Estimated $s=0.0067, \mathrm{NSR} \approx 71.6 \%$. (c) UK index. Estimated $s=0.0075$, NSR $\approx 85.2 \%$. (d) Hong Kong index. Estimated $s=0.0106$, $\mathrm{NSR} \approx 53.5 \%$. (e) Japanese index. Estimated $s=0.0095, \mathrm{NSR} \approx 50.9 \%$. (f) Singaporean index. Estimated $s=0.0076, \mathrm{NSR} \approx 44.3 \%$. 
In this study, we use local analysis (with hyper-cubes when calculating the correlation integral), and we observe the saturation on the noise level estimation for embedding dimension up to $5 .{ }^{15}$ From figure 2 we observe that the deviation of the estimated noise from the normal noise is larger in the eastern markets than it is in the western markets.

This paper arrived at two main sets of conclusions for the markets under study. Firstly, with respect to the magnitude of the noise, the western markets appear to function more efficiently than the eastern ones since the noise component appears to be proportionally much larger in the former case than in the latter. More precisely, noise to signal ratios (NSRs) were estimated at around $70 \%$ for the western markets as opposed to around $50 \%$ for the eastern ones. The results are of no surprise since the western markets are more mature and less volatile compared to the eastern ones that are, to a larger extent, influenced by speculators, foreign institutional investors and continuous regulatory changes.

Secondly, with respect to the distribution of noise, the western markets, which are considered relatively more mature and efficient, share similar characteristics with noise being distributed in a mesokurtic manner. Canadian and USA markets behave similarly in terms of the characteristics of their noise distributions while for the UK market, the high NSR nearly disables the short-term profit.

Eastern markets also have similar characteristics between them, with large noise components, which are distributed leptokurtically. In general, it can be expected that the negative or positive effects of random news and events may often be enlarged multiplicatively in such markets and this may explain the leptokurtic distribution of noise (for a relevant discussion, see, for example, Yang and Brorsen [1993]). Moreover, the leptokurtic distribution of noise in the eastern markets limits short-term predictability even though their NSRs are much lower.

\section{Conclusions}

Six international western and eastern markets (both mature and emerging ones) were considered under the nonlinear dynamics concept,

15. Szpiro (1997) followed the local analysis (with hyper-spheres when calculating the correlation integral) and used different values for the embedding dimension $\mathrm{m}$ up to 25 , until he observed saturation on the noise level estimation. 
over the period January 1, 1988 to June 30, 1999. For these markets strong evidence was found that they are not IID. This is consistent with the existing literature of other financial applications.

We applied Schreiber's method for noise estimation in time series ${ }^{16}$, since there is evidence of underlying chaotic behavior on all markets under study. We focused on the features of the stochastic part (noise) of the markets under study. Since after noise reduction we can have a better view of the time series dynamics and a smaller prediction error. Each of the markets under study consist of a one-dimensional deterministic process plus a large amount of noise. Both BDS statistic and LPCA method indicate that our markets are not consistent with the random walk hypothesis; at least for the period we study.

The western (mature) markets have NSRs of about $70 \%$ and mesokurtic noise distributions, while the eastern markets have NSRs of about $50 \%$ and leptokurtic noise distributions. We also see that the BDS statistic results are relatively large for the markets with low NSRs and relatively small for those with high NSRs. The interaction between the Canadian and US market is reflected by the distribution of noise: in both markets, the noise distribution is almost the same.

The noise standard deviation is a measure of risk, so that one can substitute the numerator of the volatility coefficient, which is the standard deviation of the time series, with the estimated noise standard deviation. Even an irregular time series derived by a low dimensional chaotic system, in the absence of noise, is considered to have no risk at all because it is completely predictable in the short run.

The noise estimation method we presented in this paper is an essentially geometric method. That is, it takes into account only the distortion of the reconstructed attractor. Evaluating the noise standard deviation, we still have no information about the noise amplification through the time evolution. An alternative substitution of the numerator of the volatility coefficient could be the noise amplification. This can be a matter for future research.

Additionally, another matter for future investigation could be the estimation of the kurtosis coefficient via our nonlinear noise estimation method. Finally, one can study both nonlinear methods presented and draw a link between the local curvature as estimated by LPCA and its effect on the noise estimation method.

16. It is generally accepted that the financial time series are characterized by heteroskedasticity. However, the existing methods (like Schreiber's for noise estimation) are still not corrected for this feature. Therefore, we can only get some rough estimation for the above quantities. In addition, the log operation we used to calculate returns, eliminates heteroskedasticity (Fialho and Pedreira ([1996]). 


\section{References}

Ashley, R.J., and Patterson, D. M. 1989. Linear versus nonlinear macroeconomics: A statistical test, International Economic Review 30 (3): $685-704$.

Abhyankar, A.; Copeland, L. S.; and Wong, W. 1995. Nonlinear dynamics in real time equity market indices: Evidence from the United Kingdom, The Economic Journal 105: 864-880.

Badii, R.; Broggi, B.; Derigetti, B.; Ravani, M.; Ciliberto, S.; Politi, A.; and Rubio, M. A. 1988. Dimension increase in filtered chaotic signals, Physical Review Letters 60: 979-982.

Baumol, W.J, and Benhabib, J. 1989. Chaos: Significance, mechanism, and economic applications, Journal of Economic Perspectives 3: 77-105.

Baumol, W.J., and Quandt, R. E. 1985. Chaos, models and their implications for forecasting, Eastern Economic Journal 11: 3-15.

Blank, S. C. 1991. "Chaos" in futures markets? A nonlinear dynamical analysis, The Journal of Futures Markets 11: 711-728.

Brock, W. A.; Dechert, W. D.; and Scheinkman, J. A. 1987. A test for independence based on the correlation dimension. Working Paper \#8702. Department of Economics, University of Wisconsin.

Brock, W. A; Hsieh, D.; and LeB aron, B. 1991. Nonlinear dynamics, chaos, and instability, statistical theory and economic evidence. Cambridge, MA: MIT Press.

Brock, W.A., and Sayers, C. 1988. Is the business cycle characterized by deterministic chaos? Journal of Monetary Economics 22: 71-90.

Broomhead, D. S.; Huke, J. P.; and Muldoon, M. R. 1992. Linear filters and non-linear systems, Journal of the Royal Statistical Society B 54: 373-382.

Broomhead, D. S.; Jones, R.; and King, G. P. 1987. Topological dimension and local coordinates from time series data, Journal of Physics A 20: L563L569.

Casdagli, M, and Eubank, S. (eds.) 1992. Nonlinear modeling and forecasting. Santa Fe Institute Studies in the Science of Complexity, Addison-W esley, Redwood City, CA.

Cecen, A. A., and Erkal, C. 1996. Distinguishing between stochastic and deterministic behavior in foreign exchange rate returns: Further evidence. Economics Letters 51: 323-329.

DeCoster, G. P.; Labys, W. C.; and Mitchell, D. W. 1992. Evidence of chaos in commodity futures prices, Journal of Futures Markets 12: 291-305.

DeGrauve, P.; Dewachter, H.; and M.Embrechts 1993. Exchange rate theory: Chaotic models of foreign exchange markets. Blackwell Editions.

Diebold, F. X., and Nason, J. A. 1990. Nonparametric exchange rate prediction? Journal of International Economics 28: 315-332.

Ding, M.; Grebogi, C.; Ott, E.; Sauer, T.; and Yorke, J. A. 1993. Plateau onset for correlation dimension: When does it occur? Physical Review Letters 70: 
3872-3875.

Eldridge, R. M.; Bernhardt, C.; and Mulvey, I. 1993. Evidence of chaos in the S\&P 500 cash index. Advances in Futures and Options Research 6: 179192.

Fialho, M., and Pedreira, C. 1996. An interval neural network architecture for time series prediction. In A. P. Refenes, Y. A. Mostafa, J. Moody, and A. Weigend (eds.) "Neural networks in financial engineering", p.p. 622-627, World Scientific.

Frank, M.; and Stengos, T. 1989. Measuring the strangeness of gold and silver rates of return, Review of Economic Studies 56: 553-567.

Fraser, A. M., and Swinney, H. 1986. Independent coordinates for strange attractors from mutual information, Physical Review A 33: 1134-1140.

Grassberger, P., and Procaccia I., 1983. Characterization of strange attractors, Physical Review Letters 50: 346-349.

Guillaume, D.M., 1995. A low-dimensional fractal attractor in the foreign exchange markets? Chapter 15. In R.R.Trippi (ed.) Chaos and nonlinear dynamics in the financial markets. Irwin Professional Publishing.

Heiner, R. A. 1983. The origin of predictable behavior, American Economic Review (September): 560-595.

Hsieh, D. 1989. Testing for nonlinear dependence in daily foreign exchange rates, Journal of Business 62: 339-368.

Hsieh, D. 1991. Chaos and nonlinear dynamics: Application to financial markets, The Journal of Finance XLVI (5): 1839-1877.

Jaditz, T., and Sayers, C. L. 1995. Nonlinearity in the interest rate risk premium. 335-357. In: R. Trippi (ed). Chaos and Nonlinear Dynamics in the Financial Markets. Irwin.

Kaen, F. R., and Rosenman, R. E. 1986. Predictable behavior in financial markets, American Economic Review (March): 212-220.

Lo, A., and MacKinley, A. C. 1989. The size and power of the variance ratio test in finite samples, Journal of Econometrics 40: 203-238.

Mayfield, E. S., and Mizrach, B. 1992. On determining the dimension of realtime stock-price data, Journal of Business \& Economic Statistics 10: 367374.

Mirowski, P., 1990. From Mandelbrot to chaos in economic theory, Southern Economic Journal, 57(2): 289-307.

Press, W.; Teukolsky, S.; Vetterling W.; and Flannery, B. 1996. Numerical Recipes in C. Cambridge University Press.

Ramsey, J.B.; Sayers, C. L.; and P.Rothman 1990. The statistical properties of dimension calculations using small data sets: Some economic applications, International Economic Review 31(4): 991-1020.

Sauer, T.; Yorke, J. A.; and Casdagli, M. 1991. Embedology, Journal of Statistical Physics 65: 579-616.

Savit, R. 1988. When random is not random: An introduction to chaos in market prices, The Journal of Futures Markets 8(3): 271-289. 
Savit, R. 1989. Nonlinearities and chaotic effects in options prices, Journal of Futures Markets 9(6): 507-518.

Scheinkman, J. A., and LeBaron, B. 1989. Nonlinear dynamics and stock returns, Journal of Business 62(3): 311-337.

Schreiber, T. 1993. Determination of the noise level of chaotic time series, Physical Review E 48: R13-R16.

Shaffer, S. 1991. Structural shifts and the volatility of chaotic markets, Journal of Economic Behavior and Organization 15(2): 201-214.

Szpiro, G. G. 1997. Noise in unspecified, non-linear time series, Journal of Econometrics 78: 229-255.

Takens, F. 1981. Detecting strange attractors in turbulence. 336-381. In: D.A. Rand, and Young, L. -S. (eds), Dynamical Systems and Turbulence. Lecture Notes in Mathematics 898. Springer-Verlang.

Theiler, J. 1986: Spurious dimension from correlation algorithms applied to limited time-series data, Physical Review A 34: 2427-2433.

Theiler, J., and Eubank, S. 1993. Don't bleach chaotic data, Chaos 3: 771-782.

Timmer, J. 1998. Modeling noisy time series: Physiological tremor, International Journal of Bifurcation and Chaos 8(7): 1505-16.

Willey, T. 1992. Testing for nonlinear dependence in daily stock indices, Journal of Economics and Business 44: 63-76.

Yang, S.-R., and Brorsen, B. W. 1993. Nonlinear dynamics of daily futures prices: Conditional heteroskedasticity or chaos? The Journal of Futures Markets 13: 175-191. 